\title{
Time Acceleration Methods for Advection on the Cubed Sphere
}

\author{
R.K. Archibald, K.J. Evans, J.B. Drake, and J.B. White III \\ Oak Ridge National Laboratory, Oak Ridge, TN
}

\begin{abstract}
Climate simulation will not grow to the ultrascale without new algorithms to overcome the scalability barriers blocking existing implementations. Until recently, climate simulations concentrated on the question of whether the climate is changing. The emphasis is now shifting to impact assessments, mitigation and adaptation strategies, and regional details. Such studies will require significant increases in spatial resolution and model complexity while maintaining adequate throughput. The barrier to progress is the resulting decrease in time step without increasing single-thread performance. In this paper we demonstrate how to overcome this time barrier for the first standard test defined for the shallow-water equations on a sphere. This paper explains how combining a multiwavelet discontinuous Galerkin method with exact linear part time-evolution schemes can overcome the time barrier for advection equations on a sphere. The discontinuous Galerkin method is a high-order method that is conservative, flexible, and scalable. The addition of multiwavelets to discontinuous Galerkin provides a hierarchical scale structure that can be exploited to improve computational efficiency in both the spatial and temporal dimensions. Exact linear part timeevolution schemes are explicit schemes that remain stable for implicit-size time steps.
\end{abstract}

\section{Introduction}

Large-scale scientific computing has maintained its exponential growth via the ever expanding parallelism while individual processor speeds have begun to stagnate 9. This trend requires the development of new algorithms that can overcome the time barrier, or effectively scale in spatial resolution while maintaining adequate throughput and accuracy. This paper takes a step towards this goal by demonstrating how the time step for advection equations on a sphere can be significantly increased by using a multiwavelet discontinuous Galerkin method with an exact linear part time-evolution scheme.

The discontinuous Galerkin (DG) method has an elegant and flexible formulation that can provide high-order accurate solutions to complicated models [5]6]. DG is a finite element method that is locally conservative and allows for an element-wise discontinuous solution approximation. DG is a scalable method because numerical information of each element is only passed locally through numerical fluxes to the nearest neighbors. In a set of papers, the DG method 
was successfully implemented on the sphere for advection models [1] and the shallow water equation [12]. We build on this work by merging multiwavelets with discontinuous Galerkin on the sphere and accelerate the time step by using an exact linear part (ELP) time-evolution scheme.

Multiwavelets are a discontinuous, orthogonal, compactly supported, multiscale set of functions with vanishing moments that yield high-order $h p$-adaptive approximations of $L^{2}$ functions 1 . Combination of multiwavelets with the DG method results in a computationally fast and effective multi-scale adaptive DG method [3]. ELP has been demonstrated to be particularly effective and efficient for multiwavelet-based schemes 24 since the operators generated for the ELP method remain sparse in a multiwavelet representation.

This paper is orgainized as follows. In section 2 we introduce the multiwavelet basis and its key features. In section 3 we describe the DG method for the cubed sphere and further demonstrate how multiwavelets are incorporated. Section 4 describes ELP for the multiwavelet DG method. Section 5 demonstrates the time acceleration of advection problems on the cubed sphere. Section 6 ends the paper with a discussion of the results.

\section{Multiwavelet Bases}

In this section we briefly summarize the important properties of the multiwavelet basis derived and developed in [1] and introduce notation as given in [2]. We begin by defining $\mathbf{V}_{n}^{k}$ as a space of piecewise polynomial functions, for $k=1,2, \ldots$, and $n=0,1,2, \ldots$, as

$$
\mathbf{V}_{n}^{k}=\left\{f: f \in \Pi_{k}\left(I_{n l}\right), \text { for } l=0, \ldots, 2^{n}-1, \text { and } \operatorname{supp}(f)=I_{n l}\right\},
$$

where $\Pi_{k}\left(I_{n l}\right)$ is the space of all polynomials of degree less than $k$ on the interval $I_{n l}=\left[2^{n} l, 2^{n}(l+1)\right]$. Using this space, we can describe not only multiwavelets, but the solution space that the DG method uses for approximation. The multiwavelet subspace $\mathbf{W}_{n}^{k}, n=0,1,2, \ldots$, is defined as the orthogonal complement of $\mathbf{V}_{n}^{k}$ in $\mathbf{V}_{n+1}^{k}$, or

$$
\mathbf{V}_{n}^{k} \oplus \mathbf{W}_{n}^{k}=\mathbf{V}_{n+1}^{k}, \quad \mathbf{W}_{n}^{k} \perp \mathbf{V}_{n}^{k}
$$

The immediate result of this definition of the multiwavelet subspace is that it splits $\mathbf{V}_{n}^{k}$ into $n+1$ orthogonal subspaces of different scales, as

$$
\mathbf{V}_{n}^{k}=\mathbf{V}_{0}^{k} \oplus \mathbf{W}_{0}^{k} \oplus \mathbf{W}_{1}^{k} \oplus \ldots \oplus \mathbf{W}_{n-1}^{k} .
$$

Given a basis $\phi_{0}, \ldots, \phi_{k-1}$ of $\mathbf{V}_{0}^{k}$, the space $\mathbf{V}_{n}^{k}$ is spanned by $2^{n} k$ functions which are obtained from $\phi_{0}, \ldots, \phi_{k-1}$ by dilation and translation,

$$
\phi_{j l}^{n}(x)=2^{n / 2} \phi_{j}\left(2^{n} x-l\right), \quad j=0, \ldots, k-1, \quad l=0, \ldots, 2^{n}-1 .
$$

By construction similar properties hold for multiwavelets. If the piecewise polynomial functions $\psi_{0}, \ldots, \psi_{k-1}$ form an orthonormal basis for $\mathbf{W}_{0}^{k}$, then by dilation and translation the space $\mathbf{W}_{n}^{k}$ is spanned by $2^{n} k$ functions

$$
\psi_{j l}^{n}=2^{n / 2} \psi_{j}\left(2^{n} x-l\right), \quad j=0, \ldots, k-1, \quad l=0, \ldots, 2^{n}-1 .
$$


A function $f \in \mathbf{V}_{n}^{k}$ can be represented by the following expansion of scaling functions.

$$
f(x)=\sum_{l=0}^{2^{n}-1} \sum_{j=0}^{k-1} s_{j l}^{n} \phi_{j l}^{n}(x),
$$

where the coefficients $s_{j l}^{n}$ are computed as

$$
s_{j l}^{n}=\int_{2^{-n} l}^{2^{-n}(l+1)} f(x) \phi_{j l}^{n}(x) d x .
$$

The decomposition of $f(x)$ has an equivalent multiwavelet expansion given by

$$
f(x)=\sum_{j=0}^{k-1}\left(s_{j 0}^{0} \phi_{j}(x)+\sum_{m=0}^{n-1} \sum_{l=0}^{2^{m}-1} d_{j l}^{m} \psi_{j l}^{m}(x)\right),
$$

with the coefficients

$$
d_{j l}^{m}=\int_{2^{-n} l}^{2^{-n}(l+1)} f(x) \psi_{j l}^{n}(x) d x .
$$

It is demonstrated in [1] how fast transforms between (6) and (8) can be developed using two-scale difference equations. Specifically, expansion coefficients of multiwavelets with $k$ vanishing moments can be constructed on consecutive levels $m$ and $m+1$ through repeated application of

$$
\begin{aligned}
s_{j l}^{m} & =\sum_{j=0}^{k-1}\left(h_{i j}^{(0)} s_{j, 2 l}^{m+1}+h_{i j}^{(1)} s_{j, 2 l+1}^{m+1}\right), \\
d_{j l}^{m} & =\sum_{j=0}^{k-1}\left(g_{i j}^{(0)} s_{j, 2 l}^{m+1}+g_{i j}^{(1)} s_{j, 2 l+1}^{m+1}\right),
\end{aligned}
$$

using the scaling coefficients $h_{i j}^{(0)}$ and $g_{i j}^{(0)}$ for $i, j=0, \ldots, k-1$. The inverse operation that takes expansion coefficients of (8) to (6) is given by

$$
\begin{aligned}
s_{j, 2 l}^{m+1} & =\sum_{j=0}^{k-1}\left(h_{j i}^{(0)} s_{j, l}^{m}+g_{j i}^{(0)} d_{j l}^{m}\right), \\
d_{j, 2 l+1}^{m+1} & =\sum_{j=0}^{k-1}\left(h_{j i}^{(1)} s_{j, l}^{m}+g_{j i}^{(1)} d_{j, l}^{m}\right),
\end{aligned}
$$

for the scaling coefficients $h_{i j}^{(0)}$ and $g_{i j}^{(0)}$, for $i, j=0, \ldots, k-1$.

The total number of expansion coefficients in (6) and (8) are the same, but the number of significant expansion coefficients for a given error tolerance level $\epsilon$ will be different. A benefit of using the multiwavelet expansion (8) is that much-fewer significant expansion coefficients are generally needed. A result of this property when multiwavelets are used in DG methods is an increase in computational speed and efficiency [1. In this paper we use hard thresholding to eliminate non-significant expansion coefficients. 


\section{Multiwavelet Discontinuous Galerkin Method on the Cube Sphere}

In this section we begin by describing the multiwavelet DG [1] method in two dimensions and finish by demonstrating how this method can be used with the cube-sphere geometry to model equations on the sphere.

Consider the two-dimensional scalar nonlinear conservation law

$$
u_{t}+\nabla \cdot f(u)=0, \text { in }[0,1]^{2} \times[0, T] .
$$

We restrict our attention to uniform Cartesian meshes since they provide the most natural representation for multiwavelets; other mesh choices are possible but the implementation becomes more challenging [7. Given a fixed order $k \geq 0$ and resolution $n \geq 0$, variational formulation of the DG method is derived by multiplying (12) by the test functions $\phi_{j l} \in \mathbf{V}_{n}^{k}$ and integrating to obtain

$$
\begin{aligned}
\int_{I_{n \ell}} \int_{I_{n l}} \frac{\partial u}{\partial t} \phi_{j l}^{n}(x) \phi_{\jmath \ell}^{n}(y) d x d y & =\int_{I_{n \ell}} \int_{I_{n l}} f(u) \frac{\partial \phi_{j l}^{n}(x)}{\partial x} \phi_{\jmath \ell}^{n}(y) d x d y \\
& +\int_{I_{n \ell}} \int_{I_{n l}} f(u) \phi_{j l}^{n}(x) \frac{\partial \phi_{\jmath \ell}^{n}(y)}{\partial y} d x d y \\
& -\int_{\partial\left[I_{n \ell} \times I_{n l}\right]} f(u) \cdot \mathbf{n} \phi_{j l}^{n}(x) \phi_{\jmath \ell}^{n}(y) d s
\end{aligned}
$$

for $j, \jmath=0, \ldots, k-1$ and $l, \ell=0,1, \ldots, 2^{n}-1$, where $\mathbf{n}$ is the outward-facing unit normal vector on the element boundary $\partial\left[I_{n \ell} \times I_{n l}\right]$. Consider the following two-dimensional multiwavelet expansion.

$$
u_{h}(x, y, t)=\sum d_{j l, \jmath \ell}^{m, \mu} \psi_{j l}^{m}(x) \psi_{\jmath \ell}^{\mu}(y),
$$

with summation taken over $j, \jmath=0, \ldots, k-1$ and $m, \mu=-1,0, \ldots, n-1$ and $l=0,1, \ldots, \min \left(0,2^{m}-1\right)$ and $\ell=0,1, \ldots, \min \left(0,2^{\mu}-1\right)$, where notation is condensed by defining $\psi_{j 0}^{-1}(\cdot) \equiv \phi_{j}(\cdot)$, for $j=0, \ldots, k-1$. The numerical multiwavelet DG scheme supplants the test functions (13) with multiwavelets and solves

$$
\begin{aligned}
\int_{I_{n \ell}} \int_{I_{n l}} \frac{\partial u_{h}}{\partial t} \psi_{j l}^{n}(x) \psi_{\jmath \ell}^{n}(y) d x d y & =\int_{I_{n \ell}} \int_{I_{n l}} f\left(u_{h}\right) \frac{\partial \psi_{j l}^{n}(x)}{\partial x} \psi_{\jmath \ell}^{n}(y) d x d y \\
& +\int_{I_{n \ell}} \int_{I_{n l}} f\left(u_{h}\right) \psi_{j l}^{n}(x) \frac{\partial \psi_{\jmath \ell}^{n}(y)}{\partial y} d x d y \\
& -\int_{\partial\left[I_{n \ell} \times I_{n l}\right]} \hat{f}\left(u_{h}\right) \cdot \mathbf{n} \psi_{j l}^{n}(x) \psi_{\jmath \ell}^{n}(y) d s
\end{aligned}
$$

where $\hat{f}\left(u_{h}\right)$ is a monotone numerical flux, the focal point for the only communication between elements. Throughout this paper we use the well known simple 


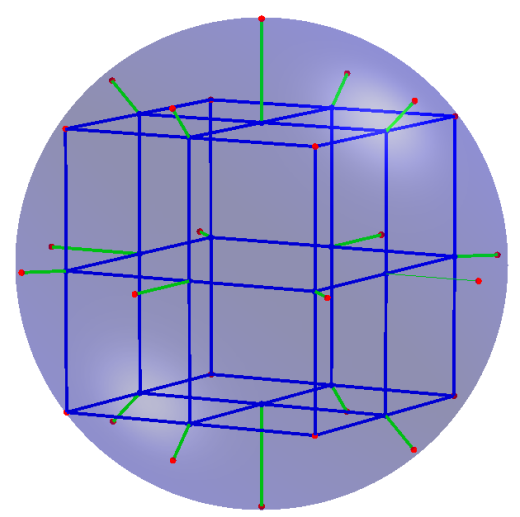

Fig. 1. The cubed-sphere gridding technique projects the red points on the sphere along radial lines to the blue points on the inscribed cube face

Lax-Friedrichs flux [10] and Gauss-Lobatto quadrature for integration. An explicit solution of (15) results directly from the orthogonality of multiwavelets, where

$$
\int_{I_{n \ell}} \int_{I_{n l}} \frac{\partial u_{h}}{\partial t} \psi_{j l}^{n}(x) \psi_{\jmath \ell}^{n}(y) d x d y=\frac{\partial d_{j l, \jmath \ell}^{m, \mu}}{\partial t}
$$

for all index values given previously.

The cubed sphere, first developed in [13, has proven to be a particularly useful gridding technique for solving partial differential equations on the sphere 111214. Figure1 1epicts the cubed sphere, where the transformation between the inscribed cube and the sphere is determined by the gnomonic (center) projection from the sphere to each face of the cube. DG is well-suited for this type of gridding [1112, since each face can be solved as a separate two-dimensional problem, with faces communicating with each other as boundary conditions.

\section{Time Discretization}

We use a method of time stepping that has been demonstrated to be particularly effective and efficient for multiwavelet schemes [24]. The idea behind the development of these schemes, as it is related to this research, is to convert differential equations of the form

$$
u_{t}=\mathcal{L} u+\mathcal{N}(u),
$$

where the system is split into a linear operator $\mathcal{L}$ and nonlinear operator $\mathcal{N}$, into the equivalent integral equation,

$$
u(t)=e^{t \mathcal{L}} u_{0}+\int_{0}^{t} e^{(t-\tau) \mathcal{L}} \mathcal{N}(u) d \tau .
$$


The multiwavelet basis allows fast scaling and squaring methods that produce sparse and highly accurate approximations to the exponential linear operator. These time-stepping schemes are therefore called exact linear part (ELP) schemes.

This paper focuses on linear advection equations on the sphere, and therefore we will only discuss how to approximate the exponential operator $e^{t \mathcal{L}}$. Suppose we are given the matrix $\mathcal{L}$ and an error tolerance $\epsilon$; the scaling and squaring method that approximates the exponential linear operator is as follows.

1. Compute the exponent $j$ such that $t\|\mathcal{L}\|_{2} / 2^{j}<\epsilon$.

2. Compute the approximation $e^{t \mathcal{L} / 2^{j}}=\mathcal{I}+t\|\mathcal{L}\|_{2} / 2^{j}$.

3. $e^{t \mathcal{L} / 2^{j}}$ is squared $j$ times to obtain $e^{t \mathcal{L}}$.

Sparsity is maintained by truncating to the error tolerance at each step.

\section{$5 \quad$ Numerical Results}

In this section we consider the following problem of advection on the sphere, a problem that has specific importance to the development of climate models.

Example 1. Given the advecting field $h$, the equation for advection in flux form is

$$
\frac{\partial h}{\partial t}+\nabla \cdot(h \mathbf{v})=0 .
$$

The first test in the standard suit developed by the climate modeling community [15] is to solve (18) on the surface of a sphere, with initial conditions given in spherical coordinates as

$$
h(r(\lambda, \theta))= \begin{cases}\frac{h_{0}}{2}\left(1+\cos \left(\frac{\pi r}{R}\right)\right) & \text { if } r<R, \\ 0 & \text { otherwise }\end{cases}
$$

for $r(\lambda, \theta)=a \arccos \left(\sin \left(\theta_{c}\right) \sin (\theta)+\cos \left(\theta_{c}\right) \cos (\theta) \cos \left(\lambda-\lambda_{c}\right)\right)$ and advecting wind

$$
\mathbf{v}=u_{0}\left(\begin{array}{l}
\cos (\theta) \cos (\alpha)+\sin (\theta) \cos (\lambda) \sin (\alpha) \\
-\sin (\lambda) \sin (\alpha)
\end{array}\right)
$$

Here the parameters are set to $a=6.37122 \times 10^{6} \mathrm{~m}, h_{0}=1000 \mathrm{~m},\left(\lambda_{c}, \theta_{c}\right)=$ $\left(\frac{3 \pi}{2}, 0\right), R=\frac{a}{3}, u_{0}=\frac{2 \pi a}{12 \text { days }}$, and $\alpha=\frac{\pi}{4}$. We note that this choice of $\alpha$ represents a particularly difficult problem, since the advecting cosine bell passes through four corners and along two edges of the cubed-sphere grid during each full revolution.

Along with the Cosine bell initial conditions (19), we will also consider the so called Gaussian hill initial conditions,

$$
h(r(\lambda, \theta))=h_{0} e^{-\frac{r}{\rho^{2}}},
$$

for $\rho=2500 \mathrm{~km}$. 


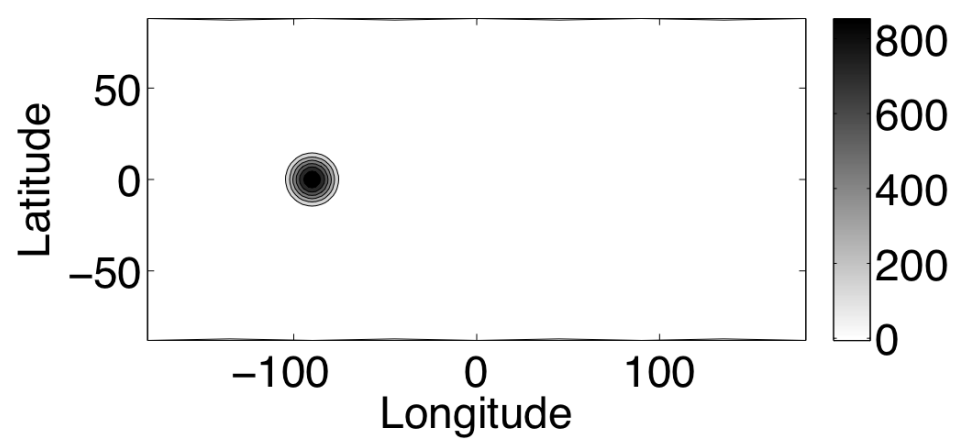

(a)

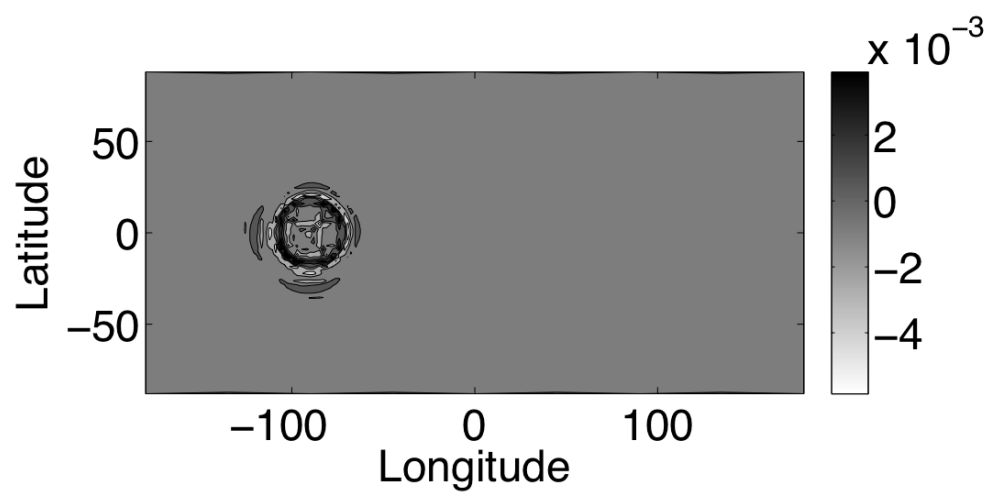

(b)

Fig. 2. (a) Final multiwavelet DG solution of Example 1 for Cosine bell initial conditions. (b) Relative error after one complete revolution, with $N=16, k=3$ and $\mathrm{CFL}=18.2$.

Throughout this section we will use

$$
\mathrm{CFL}=\frac{u_{0} \Delta t}{\Delta x} \quad \text { and } \quad N_{e}=6 N^{2}
$$

where $\Delta t$ is the time step, $N_{e}$ is the total number of elements on each cube face, and $\Delta x=\frac{1}{N}$.

Figure 2 depicts the multiwavelet DG solution and relative error of Example 1 for Cosine bell initial conditions, with $N=16, k=3$ and $\mathrm{CFL}=18.2$. It can be seen that using ELP time stepping provides a stable solution for time steps that significantly exceed the CFL requirement for explicit methods. Figure 3 depicts the same multiwavelet DG solution and relative error of Example 1 for Gaussian hill initial conditions. The difference between the multiwavelet DG solution and the exact solution is no more than a fraction of a percent for each initial condition and is considerable better for Gaussian hill initial conditions due to the increased smoothness of this initial condition. 


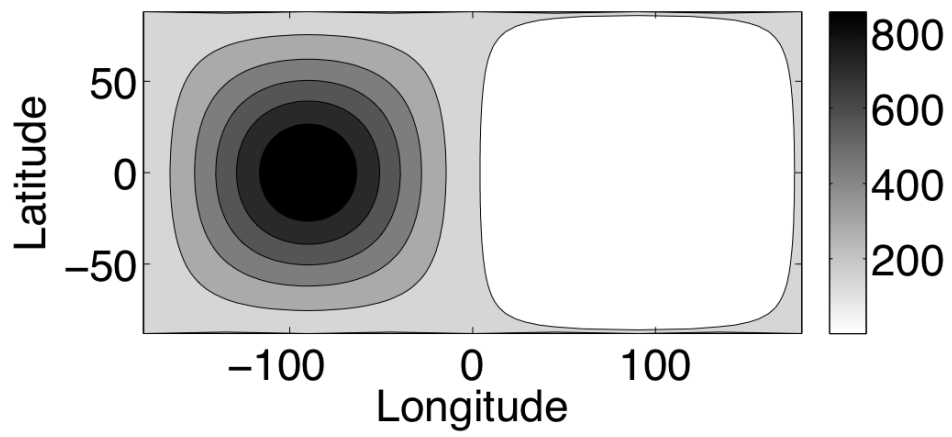

(a)

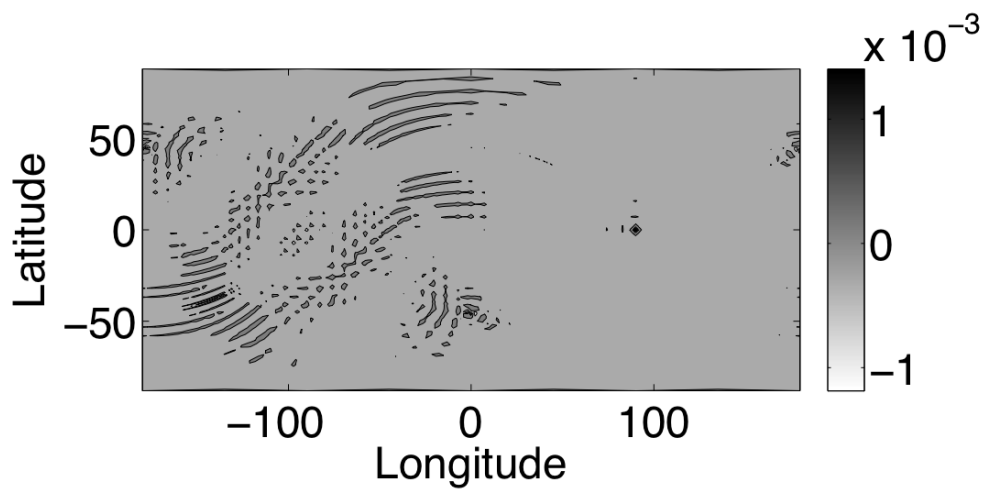

(b)

Fig. 3. (a) Final multiwavelet DG solution of Example 1 for Gaussian hill initial conditions. (b) Relative error after one complete revolution, with $N=16, k=3$ and $\mathrm{CFL}=18.2$.

Table 1 gives more-detailed insight into the properties of multiwavelet DG. We compare fourth order in time Runge-Kutta time stepping (RK4) 8 to ELP. Since Example 1 is linear, we can convert the Runge-Kutta method into an equivalent matrix operation. Each time step for the ELP method also consists of one matrix operation, and therefore we use the number of non-zero elements, $N_{z}$, in each time-evolution matrix to give a measure of the computational effort for each time step. Our first observation from Table 1 is that for both types of initial conditions the $L_{2}$ error and order of convergence is comparable for each time-stepping method and CFL number. We note that the convergence rates and errors are similar to the results published in [11 for the same problem, with cosine bell initial condition, using a DG method with CFL $=0.1$ and a third-order Runge-Kutta method. We report that in this study CFL > 0.35 resulted in instability for the RK4 method. Finally, it can be seen that the ELP method can significantly increase the time step while preserving accuracy. ELP time stepping provided a sixteen-fold acceleration of Runge-Kutta with no significant increase in the number of nonzero elements in the time-evolution matrixes. 
Table 1. Convergence rates for Example 1 using RK4 and ELP time stepping for the multiwavelet DG method with order $k=3$ and drop tolerance $\epsilon=10^{-4}$ for the ELP with $\mathrm{CFL}=4.8$ and $\epsilon=10^{-5}$ otherwise. The number of non-zero elements for each operator is give by $N_{z}$.

\begin{tabular}{|c|c|c|c|c|c|c|c|c|c|}
\hline \multirow[b]{2}{*}{$\underline{\mathrm{N}}$} & \multicolumn{3}{|c|}{ RK4 $(\mathrm{CFL}=0.3)$} & \multicolumn{3}{|c|}{$\mathrm{ELP}(\mathrm{CFL}=4.8)$} & \multicolumn{3}{|c|}{$\mathrm{ELP}(\mathrm{CFL}=18.2)$} \\
\hline & $L_{2}$ error & Order & $N_{z}$ & $L_{2}$ error & Order & $N_{z}$ & $L_{2}$ error & Order & $N_{z}$ \\
\hline \multicolumn{10}{|c|}{ cosine bell } \\
\hline 4 & $1.98 \mathrm{e}-1$ & - & $5.7 \mathrm{e} 5$ & $1.98 \mathrm{e}-1$ & - & $5.9 \mathrm{e} 5$ & $1.96 \mathrm{e}-1$ & - & $1.5 \mathrm{e} 6$ \\
\hline 8 & $4.04 \mathrm{e}-2$ & 2.30 & $2.4 \mathrm{e} 6$ & $4.18 \mathrm{e}-2$ & 2.25 & $2.5 \mathrm{e} 6$ & $4.11 \mathrm{e}-2$ & 2.26 & $8.2 \mathrm{e} 6$ \\
\hline 16 & $7.53 \mathrm{e}-3$ & 2.42 & $9.9 \mathrm{e} 6$ & $7.61 \mathrm{e}-3$ & 2.46 & $1.0 \mathrm{e} 7$ & $7.71 \mathrm{e}-3$ & 2.14 & $3.4 \mathrm{e} 7$ \\
\hline \multicolumn{10}{|c|}{ Gaussian hill } \\
\hline 4 & $2.0 \mathrm{e}-2$ & - & $5.7 \mathrm{e} 5$ & $2.01 \mathrm{e}-2$ & - & $5.9 \mathrm{e} 5$ & $2.02 \mathrm{e}-2$ & - & $1.5 \mathrm{e} 6$ \\
\hline 8 & $3.04 \mathrm{e}-3$ & 2.72 & $2.4 \mathrm{e} 6$ & $3.06 \mathrm{e}-3$ & 2.72 & $2.5 \mathrm{e} 6$ & $3.08 \mathrm{e}-3$ & 2.72 & $8.2 \mathrm{e} 6$ \\
\hline 16 & $3.6 \mathrm{e}-4$ & 3.09 & $9.9 \mathrm{e} 6$ & $3.62 \mathrm{e}-4$ & 3.08 & $1.0 \mathrm{e} 7$ & $3.63 \mathrm{e}-4$ & 3.08 & $3.4 \mathrm{e} 7$ \\
\hline
\end{tabular}

Also, a sixty-fold time acceleration was achieved at the cost of a three-fold increase in the number of nonzero elements.

\section{Conclusions}

This research has demonstrated that significant increases in time-step length are possible for advection problems on the cubed sphere by using an ELP multiwavelet DG method as compared to DG. A sixty-fold increase in time step is achieved for the first test in the standard suit developed by the climate modeling community [15] in the most-challenging advection direction for the cubed-sphere geometry. The cost of this time acceleration is a three-fold increase in the number of spatial calculations. This penalty is small relative to the gain in time acceleration and is desirable because spatial operations offer better opportunities for parallelization.

\section{Acknowledgments}

This research has been sponsored by the Laboratory Research and Development Program of Oak Ridge National Laboratory (ORNL), managed by UT-Battelle, LLC for the U.S. Department of Energy under Contract No. DE-AC05-00OR22725. Accordingly, the U.S. Government retains a non-exclusive, royalty-free license to publish or reproduce the published form of this contribution, or allow others to do so, for U.S. Government purposes.

\section{References}

1. Alpert, B.: A class of bases in $L^{2}$ for the sparse representation of integral operators. SIAM J. Math. Anal. 24(1), 246 (1993)

2. Alpert, B., Beylkin, G., Gines, D., Vozovoi, L.: Adaptive solution of partial differential equations in multiwavelet bases. Journal of Computational Physics 182(1), 149 (2002) 
3. Archibald, Fann, Shelton: Adaptive Discontinuous Galerkin Methods in Multiwavelets Bases. Journal of Scientific Computing (2008) (submitted)

4. Beylkin, G., Keiser, J.M., Vozovoi, L.: A new class of stable time discretization schemes for the solution of nonlinear PDEs. Journal of Computational Physics 147, 362 (1998)

5. Cockburn, B., Shu, C.W.: The local discontinuous Galerkin method for timedependent convection diffusion systems. SIAM Journal on Numerical Analysis 35, 2440 (1998)

6. Cockburn, B., Shu, C.W.: Runge-Kutta discontinuous Galerkin methods for convection-dominated problems. Journal of Scientific Computing 16(3), 173 (2001)

7. Coult, N.: Introduction to Discontinuous Wavelets. In: Cockburn, B., Karniadakis, G.E., Shu, C.-W. (eds.) Discontinuous Galerkin Methods: Theory Computation and Applications. Springer, Heidelberg (2000)

8. Davis, P.J., Polonsky, I.: Numerical Interpretation, Differentiation, and Integration. In: Abramowitz, M., Stegun, I. (eds.) Handbook of Mathematical Functions. Dover (1972)

9. Drake, Jones, Vertenstein, White III, Worley: Software Design for Petascale Climate Science. In: Bader, D. (ed.) Petascale Computing: Algorithms and Applications. Chapman \& Hall/CRC, Boca Raton (2008)

10. LeVeque, R.J.: Numerical Methods for Conservation Laws. Birkhauser Verlag, Basel (1990)

11. Nair, R.D., Thomas, S.J., Loft, R.D.: A discontinuous Galerkin transport scheme on the cubed sphere. Monthly Weather Review 133(4), 814 (2005)

12. Nair, R.D., Thomas, S.J., Loft, R.D.: A discontinuous Galerkin global shallow water model. Monthly Weather Review 133(4), 876 (2005)

13. Sadourny, R.: Conservative Finite-Difference Approximations of the Primitive Equations on Quasi-Uniform Spherical Grids. Monthly Weather Review 100(2), 136-144 (1972)

14. Taylor, M.A., Tribbia, J.J., Iskandrani, M.: The spectral element method for the shallow water equations on the sphere. Journal of Computational Physics 130, 92-108 (1997)

15. Williamson, D.L., Hack, J.J., Jakob, R., Swarztrauber, P.N., Drake, J.B.: A standard test set for numerical approximations to the shallow water equations in spherical geometry. Journal of Computational Physics 102, 211 (1992) 